\title{
An Alternative Anionic Polyelectrolyte for Aqueous PEDOT Dispersions: Towards Printable Transparent Electrodes
}

\author{
Anna I. Hofmann [a], Wiljan T. T. Smaal[a], Muhammad Mumtaz ${ }^{[a]}$, Dimitrios Katsigiannopoulos ${ }^{[a]}$, Cyril \\ Brochon $^{[a]}$, Falk Schütze ${ }^{[b]}$, Olaf R.Hild ${ }^{[b]}$, Eric Cloutet ${ }^{\star}[a]$ and Georges Hadziioannou ${ }^{\star[a]}$
}

\begin{abstract}
Organic conducting polymers are promising electrode materials for printable organic electronics. One of the most studied conducting polymers is PEDOT:PSS which is sufficiently conductive and transparent, but which shows some drawbacks, such as hygroscopy and acidity. In this communication we present a new approach to stabilize PEDOT in aqueous dispersions by replacing the PSS with a new basic polyanion based on a polystyrene backbone with (trifluoromethylsulfonyl)imide (TSFI) side groups. The PEDOT:PSTFSIK dispersions were obtained by oxidative polymerization of EDOT in aqueous PSTFSIK solution and were characterized regarding their composition, morphology, doping, rheological behavior and opto-electronic performance. These PEDOT:PSTFSIK dispersions show excellent printability and good opto-electronic performance $\left(2380 \mathrm{hm} . \mathrm{sq}^{-1}\right.$ at $91 \%$ transmittance, $\sigma>260$ S. $\mathrm{cm}^{-1}$ ) and were successfully integrated as flexible electrodes in OLED and OPV devices.
\end{abstract}

Organic conducting polymers are of increasing scientific interest and are promising candidates for various applications, such as transparent and flexible electrodes in light emitting diodes[1,2], photovoltaics[3,4] and sensors[5], as antistatic coatings[6] or as active material in electrochromic devices[7,8]. The main advantages of conducting polymers are their chemical and mechanical stability, their biocompatibility as well as their processability at moderate temperatures and on large areas. One of the most studied conducting polymers is PEDOT:PSS (Poly(3,4-ethylenedioxythiophene) Polystyrene sulfonate). Due to its relatively high conductivity and transparency in the doped state it has been used for a large variety of applications [1-8]. Nevertheless, as mentioned in many reports, it shows some drawbacks, such as hygroscopy, acidity and poor wetting behavior on organic layers. In this communication we present a new approach to stabilize PEDOT in aqueous dispersions by replacing the polystyrenesulfonic acid with a new, basic anionic polymer, based on (trifluoromethylsulfonyl)imide (TSFI) side groups attached to a polystyrene backbone. The PEDOT:polyanion systems were characterized regarding their morphology, composition, doping and opto-electronic performance. Furthermore the effect of polymerization

[a] Laboratoire de Chimie des Polymères Organiques (LCPO), Université de Bordeaux, CNRS UMR 5629, 16 Avenue Pey-Berland, F-33607 Pessac Cedex, France *cloutet@enscbp.fr and hadzii@enscbp.fr

[b] Center for Organic Materials and Electronic Devices Dresden (COMEDD) , Fraunhofer FEP, Maria-Reiche-Straße 2, 01109 , Dresden, Germany

Supporting information for this article is given via a link at the end of the document conditions on the conductivity of the resulting PEDOT:polyanion was investigated. These PEDOT:polyanion dispersions showed excellent printability and good opto-electronic performance (2380hm. $\mathrm{sq}^{-1}$ at $91 \%$ transmittance, $\sigma>260 \mathrm{~S} . \mathrm{cm}^{-1}$ ) and were successfully integrated in OLED and OPV devices.

Polyelectrolytes, such as the polyanion in this work, are known for their application in lithium batteries [9], but they can also act as counter ion of doped conducting polymers and therefore stabilize them in aqueous dispersion. In the continuation of our work on PEDOT dispersions [10], we chose to stabilize PEDOT using a poly[4-styrenesulfonyl (trifluoromethyl sulfonyl) imide potassium salt], PSTFSIK. Even though the synthesis of such polyelectrolytes was pioneered by Armand and coll.[9] we developed a controlled polymerization route, using a reversible Addition-Fragmentation Chain Transfer (RAFT) process resulting in a well-defined, water soluble polymer in a wide range of molar masses from $20 \mathrm{kDa}$ to $500 \mathrm{kDa}$ together with low dispersity $(D<1.4)$. (ref manuscript submitted)

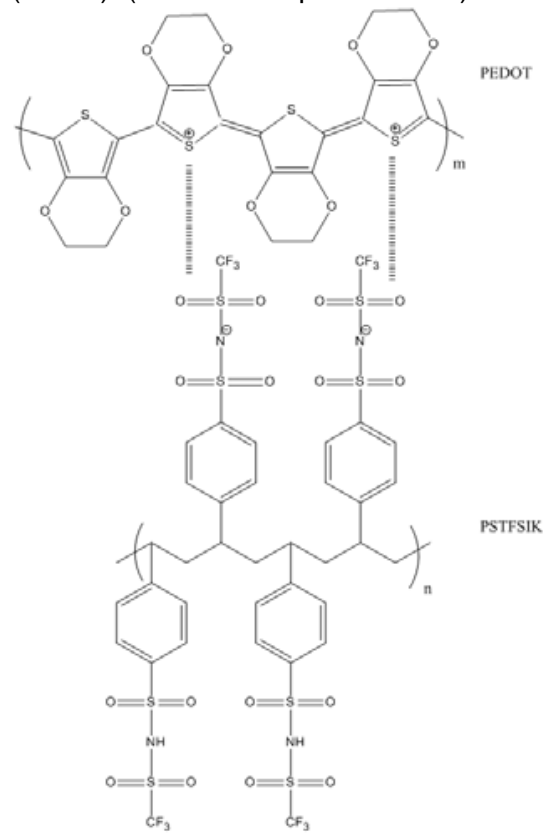

Figure 1. Schematic of the PEDOT:PSTFSIK complex. The negatively charged units of the PSTFSIK stabilize positive charge carriers on the PEDOT chains.

The PEDOT:Polyanion (PEDOT:PSTFSIK) dispersions were obtained by oxidative polymerization of EDOT in aqueous PSTFSIK solution (see experimental part). Figure 1 displays the chemical structure of PEDOT:PSTFSIK. For the sake of comparison, a PEDOT:PSS dispersion was synthesized and purified with the same procedure as the PEDOT:PSTFSIK. 
As illustrated in figure 2, the polymerization of EDOT was evidenced by UV/Vis spectroscopy. The concentration and "doping" level of PEDOT in the dispersion can be followed by monitoring the absorption intensities at $500 \mathrm{~nm}, 900 \mathrm{~nm}$ and above $900 \mathrm{~nm}$, which are generally assigned to neutral, polaronic and bipolaronic states of PEDOT, respectively[11,12]. At the beginning of the polymerization reaction the absorption for wavelengths higher than 450nm increased steadily, indicating a growing PEDOT concentration in the dispersion. At longer time scales only the absorption peak at $900 \mathrm{~nm}$ kept rising, indicating the continuous formation of polaronic states. This change in electronic configuration of PEDOT can be experimentally linked to the opto-electronic performance of the dry PEDOT:PSTFSIK films. The influence of the reaction time, along with the effect of the temperature and the $\mathrm{pH}$ on the properties of PEDOT:PSTFSIK have been investigated and will be discussed later in this manuscript. After the polymerization, the resulting inks were purified by ultrafiltration to remove remaining reactants and oxidants.
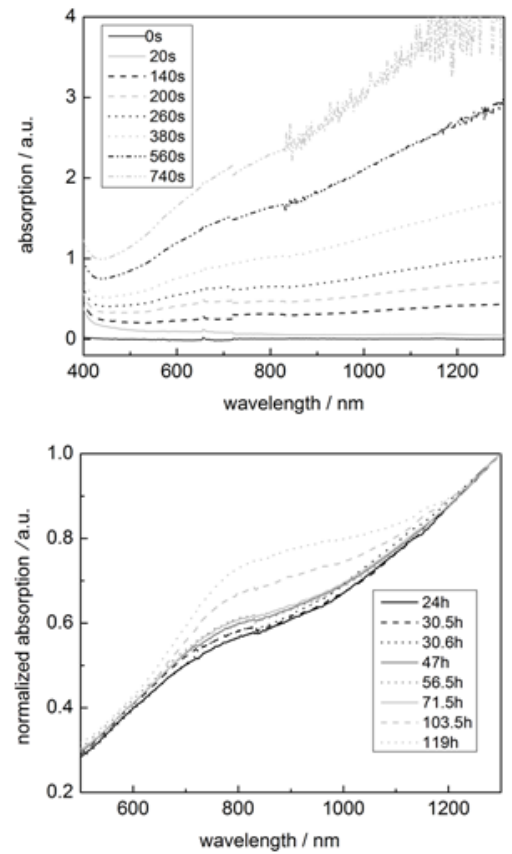

Figure 2. Evolution of the UV/Vis absorption spectrum during the EDOT polymerization over time for $0 \mathrm{~s}$ to $740 \mathrm{~s}$ (above) and for $24 \mathrm{~h}$ to $115 \mathrm{~h}$ of synthesis.

After purification all samples showed a pH between 2.1 and 3.5 (home made PEDOT:PSS pH 2.0) and a concentration of $0.7 \mathrm{wt} \%$ in water (see section "experimental" and figure S1 in supporting information).

Thermal analysis such as TGA and DSC measurements proved that PEDOT:PSTFSIK is stable up to $180^{\circ} \mathrm{C}$ and did not reveal any glass transition or crystallization temperatures (see figure S2 and S3 in supporting information).

In order to determine the molar ratio of PSTFSIK to PEDOT in the PEDOT:PSTFSIK bulk, fluorine quantification via ICP-MS analysis was performed. The PSTFSIK to PEDOT ratio was found to be 0.9. There are no comparable measurements for PEDOT:PSS, as it does not possess an element which is exclusively present in PSS and which is therefore easily traceable, such as fluorine in PSTFSIK. The comparison of the sulfur peaks in the X-ray photoelectron spectroscopy (XPS) S2p spectrum of home-made and commercial PEDOT:PSS (Clevios $\mathrm{PH} 1000$ ) revealed a PSS to PEDOT ratio of 3.0 and 2.3, respectively. These values are similar to the PSS concentration that is found by XPS measurements for commercial PEDOT:PSS in literature [13-15]. In comparison, the PSTFSIK to PEDOT ratio in PEDOT:PSTFSIK was found to be between 2.6 and 3 (XPS Sp2 spectra in figure S4 supporting information). We observed that the PEDOT content in PEDOT:PSTFSIK measured by XPS was systematically lower than the one measured by ICP-MS. Taking into account that XPS is a surface sensitive method, whereas ICP-MS gives access to the bulk composition, we can state that the PEDOT content on the PEDOT:PSTFSIK film surface is lower than in the bulk. The same behavior is found for PEDOT:PSS films that show a lower PEDOT concentration at the film surface than in the bulk. This is commonly explained by a preferential phase separation of PEDOT and the PSS with the PSS segregating to the film surface [16].

The morphology of PEDOT:PSTFSIK in dispersion was investigated by Transmission Electron Microscopy (TEM) (see figure 3). The TEM image displays a network structure of interconnected particles with an average size of $150 \mathrm{~nm}$. In contrast, home-made PEDOT:PSS shows separated agglomerates with diameters up to $1 \mu \mathrm{m}$ (see figure S5 in supporting information). Interestingly, the addition of a cosolvent like DMSO changes the solubility parameters and thus affects the colloidal morphology (see S5).

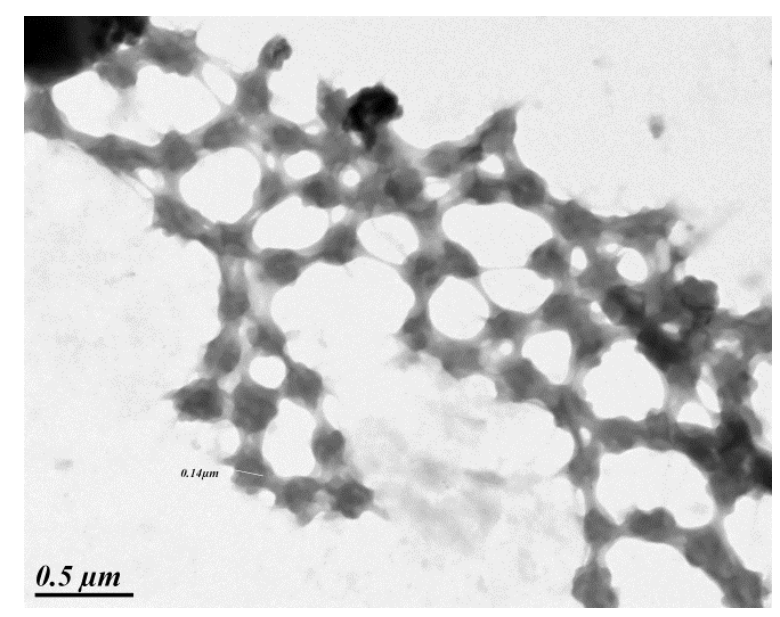

Figure 3 TEM image of PEDOT:PSTFSIK 250kDa cast from aqueous dispersion of $0.007 \mathrm{wt} \%$ in water.

Further information on the dispersion was obtained via rheological measurements. Already at low concentrations of about $1 \mathrm{wt} \%$ in water the PEDOT:PSTFSIK and the home-made PEDOT:PSS are highly viscous and show shear thinning behavior (see figure S6 supporting information). In comparison, the Clevios $\mathrm{PH} 1000$ is less viscous and shows Newtonian behavior at intermediate shear rates. In oscillating shear experiments on PEDOT:PSTFSIK demonstrates the 
characteristics of a gel (G'> G'), whereas Clevios PH1000 shows the behavior of a viscoelastic liquid (see figure S7 supporting information). The gel like behavior, the high viscosity and shear thinning properties of the PEDOT:PSTFSIK inks facilitate the processing via doctor blading or bar coating on large areas.

The opto-electronic properties of the PEDOT:PSTFSIK films were determined by measuring their sheet resistance and transmittance. To allow a valid comparison of the film properties independent of the film thickness, a figure of merit $\sigma_{D C} / \sigma_{o p t}$ is calculated using the following equation (1). [17]

Equation 1

$$
T(\lambda=550 \mathrm{~nm})=\left(1+\frac{188.5}{R} \frac{\sigma_{\text {opt }}}{\sigma_{D C}}\right)^{-2}
$$

The opto-electronic performance of the different PEDOT systems is presented in table S1 in the supporting information. The home-made PEDOT:PSS shows similar conductivity as the PEDOT:PSTFSIK $\left(224 \pm 20 \mathrm{Scm}^{-1}\right.$ for PSTFSIK of $\left.250 \mathrm{kDa}\right)$ but a 2.7 times smaller figure of merit. This is due to a lower absorption coefficient of the PEDOT:PSTFSIK compared to PEDOT:PSS $\left(2 \cdot 10^{-3} \mathrm{~cm}^{-1}\right.$ and $5 \cdot 10^{-3} \mathrm{~cm}^{-1}$, respectively). Nevertheless, both PEDOT:PSTFSIK ( $\sigma$ ranging from 96 to $224 \mathrm{Scm}^{-1}$ for a series of samples, $\left.\sigma_{\mathrm{DC}} / \sigma_{\mathrm{opt}}=13 \pm 3\right)$ and homemade PEDOT:PSS $\left(\sigma=229 \mathrm{Scm}^{-1} \pm 34 \mathrm{Scm}^{-1}, \sigma_{\mathrm{DC}} / \sigma_{\mathrm{opt}}=6\right)$ show lower conductivity and a smaller figure of merit than the Clevios $\mathrm{PH} 1000\left(\sigma=778 \mathrm{Scm}^{-1} \pm 116 \mathrm{Scm}^{-1}, \sigma_{\mathrm{DC}} / \sigma_{\mathrm{opt}}=24 \pm 5\right)$. The electronic properties of PEDOT:PSTFSIK films, stored under ambient conditions, were stable over months (see figure S9 in supporting information)

All the above measured properties were obtained for PEDOT:PSTFSIK dispersions in which $5 \mathrm{wt} \%$ DMSO was added It is well known that the addition of high boiling point solvents like DMSO to PEDOT:PSS drastically increases the conductivity of the films $[1,18,19]$. The same effect is observed for PEDOT:PSTFSIK inks. For PEDOT:PSTFSIK a DMSO concentration of $5 \mathrm{wt} \%$ increases the film performance up to 80 times compared to the pristine ink, while higher concentrations of DMSO do not further improve the performance (see figure S8 supporting information) and eventually cause the formation of inhomogeneities during film deposition.

The effect of some key polymerization parameters on the optoelectronic performance of the resulting dry PEDOT:PSTFSIK films was studied. Initial experiments indicated that the $\mathrm{pH}$ of the synthesis medium has a significant impact on the polymerization of EDOT and on the conductivity of the resulting PEDOT:PSTFSIK films (see figure 4). In acidic conditions the reaction is accelerated, whereas the addition of a base completely inhibits the polymerization (see experimental part). In aprotic solvents such as DMSO, the polymerization of EDOT does not take place. This is in agreement with the mechanism for the oxidative polymerization of thiophenes described by Higashimura et al. [20] in which the transfer of protons to the solvent is a crucial step. The performance of PEDOT:PSTFSIK synthesized in acidified solutions was much lower than the performance of PEDOT:PSTFSIK made in the basic PSTFSIK solution.
The second important parameter that was studied is the temperature of polymerization. We observed that by lowering the polymerization temperature, the figure of merit of the PEDOT:PSTFSIK films was increased (see figure 4). A decrease in temperature results in slower reaction kinetics, favoring better colloidal stability and good opto-electronic performance.

Figure 4. Performance of PEDOT:PSTFSIK films as a function of reaction

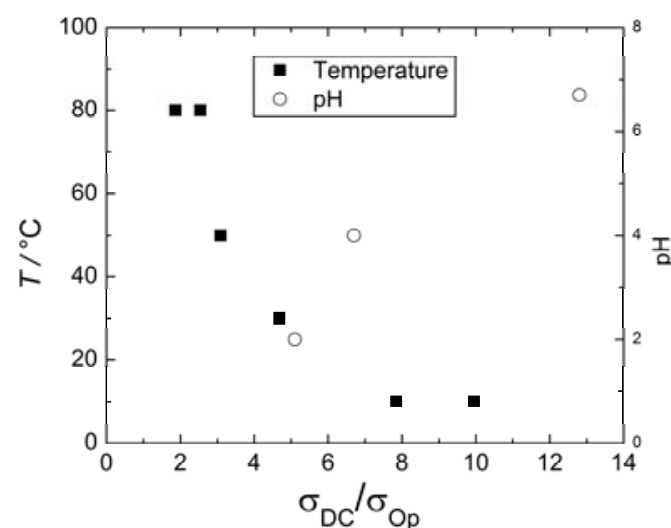

temperature and as a function of the $\mathrm{pH}$ of the PSTFSIK solution $(\mathrm{pH}$ values are controlled by the addition of $\mathrm{HCl}$ ).

A study of the relation between polymerization time and the opto-electronic properties of the films revealed that the highest performance is obtained after a reaction duration of $50 \mathrm{~h}$ at $14^{\circ} \mathrm{C}$ (see figure S10) when an optimum polaron/bipolaron ratio is reached.

The relation between polymerization conditions, molar masses, $\mathrm{pH}$ and performance are under investigation and will be the topic of a forthcoming paper.

In order to demonstrate the potential of these new conductive inks as transparent electrodes, flexible orange and white PINOLED and organic photovoltaic devices have been studied. Typical LIV curves of the orange PIN-OLEDs based on PEDOT:PSTFSIK electrodes are presented in Figure $5 \mathrm{~b}$. A total number of 40 devices were made (yield $=97.5 \%$ ) with an average efficiency of $12 \pm 1 \mathrm{CdA}^{-1}$ and $14 \pm 2 \mathrm{ImW}^{-1}$ versus a reference device using an ITO anode showing $15 \pm 7 \mathrm{cdA}^{-1}$ and $17 \pm 9 \mathrm{lmW}^{-1}$ (measurements at $1000 \mathrm{cdm}^{-2}$ ). Lifetime measurements have not yet been performed but devices encapsulated with barrier foil are stable for over several weeks under ambient conditions ( $\left.\mathrm{RH}=50-80 \%, \mathrm{~T}=15-30^{\circ} \mathrm{C}\right)$. The white PIN-OLEDs showed efficiencies of $39 \pm 5 \mathrm{cdA}^{-1}$ and $25 \pm 5 \mathrm{ImW}^{-1}$. In comparison, the PEDOT:PSS reference device reached similar efficiencies of $36 \pm 4 \mathrm{cdA}^{-1}$ and $24 \pm 31 \mathrm{~mW}^{-1}$. 

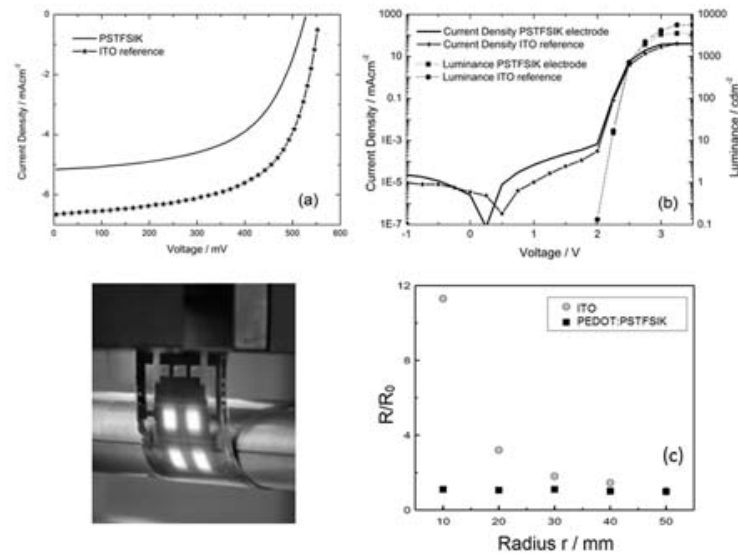

Figure 5. IV characteristics of an OPV(a) and $\operatorname{OLED(b)~device~together~with~}$ their respective reference. Devices (fabricated on $50 \times 35 \mathrm{~mm}$ PET foil) withstand flexibility tests down to a bending radius of $10 \mathrm{~mm}(\mathrm{c})$.

Figure 5a shows typical OPV IV curves of a device with PEDOT:PSTFSIK electrode (solid line) in comparison to an ITO reference device (dashed line). The difference in $V_{o c}$ is small (5\%) however the PSTFSIK based electrode is causing a decrease in the $I_{s c}(20 \%)$ with respect to the reference. This is most likely due to the higher light absorption of the polymer anode. For a total of 36 PEDOT:PSTFSIK devices (yield 86.1\%), the average power conversion efficiency was $1.8 \%$, compared to $2.6 \%$ for ITO OPV reference devices.

In conclusion, a conducting and stable PEDOT:PSTFSIK dispersion was synthesized by using a new anionic polymer for the stabilization of PEDOT. The PEDOT:PSTFSIK films show a conductivity up to $224 \mathrm{Scm}^{-1}$, which is comparable to the conductivity of home-made PEDOT:PSS, and a figure of merit up to 16 , which is about triple of the value found for our homemade PEDOT:PSS. The gel like ink is easily processable by doctor blading and the films are stable over several months under ambient conditions. The films were successfully integrated as transparent electrodes in flexible OPV (conversion efficiency of $1.8 \%)$ and OLED devices $\left(39 \pm 5 \mathrm{cdA}^{-1}, 25 \pm 5 \mathrm{lmW}^{-1}\right)$. Their performance, together with their intrinsic stability, printability and compatibility with state of the art OLED and OPV materials, show the potential of PEDOT:PSTFSIK for printable electronics.

\section{Experimental Section}

Synthesis of the PSTFSIK: The PSTFSIK was synthesized via reversible addition-fragmentation chain-transfer (RAFT) polymerization by mixing, according to the target molar mass, the proper quantity of monomer, chain transfer agent (CTA, see structure in figure S11) and azobisisobutyronitrile (AIBN) in dimethylformimide. After several freezethaw cycles, the mixture was left to polymerize at $65^{\circ} \mathrm{C}$ from several hours to few weeks, depending on the molar mass that should be obtained. The polymer was ready after precipitation in tetrahydrofuran (THF), filtration, washing with THF and drying in vacuum oven at $65^{\circ} \mathrm{C}$ for at least one day.
Synthesis of PEDOT:PSTFSIK complex: EDOT was dispersed in an aqueous solution of PSTFSIK under nitrogen atmosphere (concentration $8.18 \mathrm{mg} \cdot \mathrm{ml}^{-1}, 14 \mathrm{~mol} \cdot \mathrm{ml}^{-1}$ EDOT). $\mathrm{NH}_{4} \mathrm{~S}_{2} \mathrm{O}_{8}$ and $\mathrm{FeCl}_{3}$ at a molar ratio of 3.5 were used as oxidants. After $48 \mathrm{~h}$ at $14^{\circ} \mathrm{C}$ the polymer was washed in an ultrafiltration unit in two steps with an $\mathrm{HCl}$ solution $(\mathrm{pH} 1)$ and demineralized water.

Films were deposited by doctor blading (Erichsen coatmaster 510) on glass or PET substrates.

To measure the $\mathrm{pH}$ of the PEDOT:PSTFSIK dispersions, a Hanna instruments $\mathrm{pH}$ meter type $\mathrm{HI} 3220$ was used.

The total mass content of the dispersions was determined by TGA measurements with a TA instruments TQ50.

Fluorine analysis by inductive coupled plasma with mass spectrometer (ICP-MS) was performed at Arkema Lacq on a DX500 with an AS11 HC column. The sample was prepared by the Schoeniger oxidation method.

The sheet resistance was measured with a 4point probe (Lucas labs stand S302 with SP4 probe head).

The transmittance was determined with a Shimadzu UV3600 UV/VIS/NIR spectrometer by measuring the transmittance at 550nm.

All AFM images were obtained with a Brucker DimesionFastScan in the tapping mode with a probe with a silicon tip.

Transmission electron microscopy (TEM) was done with a Hitachi $\mathrm{H} 7650$ with an acceleration voltage of $80 \mathrm{kV}$ and a magnification of 50000 .

The OLED and OPV devices are all fabricated with a formulation based on 250kDa PEDOT:PSTFSIK in water containing 5vol\% of DMSO. The electrodes are deposited on $50 \times 35 \mathrm{~mm}$ PET substrates using doctor blading and all of them had a figure of merit $\sigma_{D C} / \sigma_{o p t}$ of $11 \pm 2$ with transparencies between 70 and $85 \%$. After deposition the electrodes are patterned by laser ablation and further layers are deposited by vacuum evaporation techniques. As substrate and for encapsulation barrier films were used.

\section{Acknowledgements}

The authors are thankful to the German Federal Ministry of Education and Research (BMBF) and the French Agence Nationale de la Recherche (ANR) for funding joint program Inter Carnot Fraunhofer called 'IMAGE' (FKZ 01SF0804). This work was also supported by the LabEx AMADEus (ANR-10-LABX-42) in the framework of IdEx Bordeaux (ANR-10-IDEX-03-02), i.e. the Investissements d'Avenir programme of the French government managed by the Agence Nationale de la Recherche. The authors thank Arkema for the CIFRE PhD fellowship (\# 2013/0352) allocated to A.I.H..

Keywords: Conducting Polymer Dispersions $\bullet$ Printed Organic Electronics • Electrodes • Flexible OLED • Flexible OPV 
[1] [1] X. Crispin, F. L. E. Jakobsson, A. Crispin, P. C. M. Grim, P. Andersson, A. Volodin, C. van Haesendonck, M. Van der Auweraer, W. R. Salaneck, M. Berggren, Chem. Mater. 2006, 18, 4354-4360.

[2] S. K. Hau, H.-L. Yip, J. Zou, A. K.-Y. Jen, Org. Electron. 2009, 10, 1401-1407.

[3] E. Ahlswede, W. Mühleisen, M. W. bin Moh Wahi, J. Hanisch, M. Powalla, Appl. Phys. Lett. 2008, 92, 143307-143307-3.

[4] R. Po, C. Carbonera, A. Bernardi, F. Tinti, N. Camaioni, Sol. Energy Mater. Sol. Cells 2012, 100, 97-114.

[5] D. Khodagholy, J. Rivnay, M. Sessolo, M. Gurfinkel, P. Leleux, L. H. Jimison, E. Stavrinidou, T. Herve, S. Sanaur, R. M. Owens, et al., Nat. Commun. 2013, 4, DOI 10.1038/ncomms3133.

[6] F. Jonas, J. T. Morrison, Synth. Met. 1997, 85, 1397-1398.

[7] J. Kawahara, P. A. Ersman, I. Engquist, M. Berggren, Org. Electron. 2012, 13, 469-474.

[8] P. Tehrani, L.-O. Hennerdal, A. L. Dyer, J. R. Reynolds, M. Berggren, J. Mater. Chem. 2009, 19, 1799.

[9] R. Meziane, J.-P. Bonnet, M. Courty, K. Djellab, M. Armand, Electrochimica Acta 2011, 57, 14-19.

[10] M. Mumtaz, A. De Cuendias, J.-L. Putaux, E. Cloutet, H. Cramail, Macromol. Rapid Commun. 2006, 27, 1446-1453.
[11] N. Massonnet, A. Carella, O. Jaudouin, P. Rannou, G. Laval, C. Celle J.-P. Simonato, J. Mater. Chem. C 2014, 2, 1278-1283.

[12] S. Garreau, J. L. Duvail, G. Louarn, Synth. Met. 2001, 125, 325-329.

[13] X. Crispin, S. Marciniak, W. Osikowicz, G. Zotti, A. W. D. van der Gon, F. Louwet, M. Fahlman, L. Groenendaal, F. De Schryver, W. R. Salaneck, J. Polym. Sci. Part B Polym. Phys. 2003, 41, 2561-2583.

[14] G. Zotti, S. Zecchin, G. Schiavon, F. Louwet, L. Groenendaal, X Crispin, W. Osikowicz, W. Salaneck, M. Fahlman, Macromolecules 2003, 36, 3337-3344

[15] T.-W. Lee, Y. Chung, Adv. Funct. Mater. 2008, 18, 2246-2252.

[16] G. Greczynski, T. Kugler, M. Keil, W. Osikowicz, M. Fahlman, W. R. Salaneck, J. Electron Spectrosc. Relat. Phenom. 2001, 121, 1-17.

[17] K. Ellmer, Nat. Photonics 2012, 6, 809-817.

[18] S.-I. Na, S.-S. Kim, J. Jo, D.-Y. Kim, Adv. Mater. 2008, 20, 4061-4067.

[19] J. Gasiorowski, R. Menon, K. Hingerl, M. Dachev, N. S. Sariciftci, Thin Solid Films 2013, 536, 211-215.

[20] H. Higashimura, S. Kobayashi, in Encycl. Polym. Sci. Technol., John Wiley \& Sons, Inc., 2002 


\section{COMMUNICATION}

Flexible and transparent

PEDOT:polyanion electrodes:

A new, water soluble family of polyanions are used to stabilize PEDOT in aqueous dispersion. The highly viscous PEDOT:polyanion dispersions can be easily processed into thin, conductive and transparent films, which can be integrated as transparent electrode in flexible organic electronic devices.

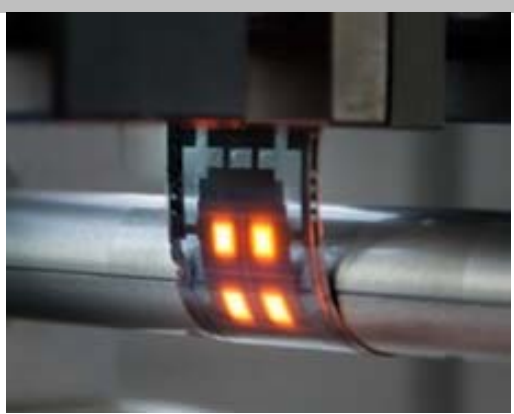

Author(s), Corresponding Author(s)*

Page No. - Page No.

Title 